\title{
ASSESSMENT OF EATING BEHAVIOURS IN ADULT RESIDENTS OF GREECE AND POLAND - AN ORIGINAL RESEARCH
}

\author{
Elżbieta Szczepańska ${ }^{1}$, Joanna Rzepecka², Aleksandra Góra², \\ Karolina Janion ${ }^{3}$,Katarzyna Urbańczyk ${ }^{2}$ \\ ${ }^{1}$ Department of Human Nutrition, School of Health Sciences in Bytom, \\ Medical University of Silesia in Katowice, Poland \\ ${ }^{2}$ Scientific Society of Young Educators, Department of Human Nutrition, \\ School of Health Sciences in Bytom, Medical University of Silesia in Katowice, Poland \\ ${ }^{3}$ Department of Nutrition-Related Disease Prevention, Department of Metabolic Disease \\ Prevention, School of Health Sciences in Bytom, Medical University of Silesia in Katowice, Poland
}

\begin{abstract}
Background: The length and the quality of life are determined by, among other things, environmental factors. The place of residence is associated with certain differences in nutritional culture, with food traditions and customs being of great importance. Also, the location of a given country in a specific climate zone has an impact on the choice of food products and their preparation.

Objective: The aim of the study was to analyse eating behaviours among adult residents of Greece and Poland as well as to investigate to what extent these behaviours are impacted by the place of residence of respondents.

Materials and methods: A total of 633 randomized respondents at the age of 50 or older (312 Greeks and 321 Poles) were included in the study. The author's questionnaire was used in the research.

Results: A total of $41.07 \%$ of respondents, including $31.09 \%$ of Greeks and $50.78 \%$ of Poles, declared having $4-5$ meals per day. Fish was consumed 1-2 times a week by $29.27 \%$ of respondents, including $23.40 \%$ of Greeks and $15.26 \%$ of Poles. Whole wheat bread was consumed several times daily by $20.7 \%$ of respondents, including $19.55 \%$ of Greeks and $21.81 \%$ of Poles. Coarse groats and pasta were consumed a few times weekly by $23.06 \%$ of respondents, including $25.64 \%$ of Greeks and $20.56 \%$ of Poles.

Conclusions: Eating behaviours in adult Poles and Greeks are varied, with healthier eating habits in the latter group. There are significant differences between the intake of food products rich in fibre and omega-3 fatty acids by residents of Greece and Poland.
\end{abstract}

Key words: eating behaviours, frequency of consumption, Poland, Greece

\section{STRESZCZENIE}

Wprowadzenie: Długość życia i jego jakość uwarunkowana jest m.in. czynnikami środowiskowymi. Miejsce zamieszkania cechuje pewna odmienność kultury związanej z żywieniem, istotne znaczenie mają w tym przypadku tradycje i zwyczaje żywieniowe. Położenie kraju w określonej strefie klimatycznej wpływa także na dobór produktów spożywczych oraz sposób przygotowania ich do spożycia.

Cel: Analiza wybranych zachowań żywieniowych dorosłych mieszkańców Grecji i Polski oraz zbadanie w jakim stopniu miejsce zamieszkania ma wpływ na występowanie różnic w zachowaniach żywieniowych osób badanych.

Material i metody: W badaniu uczestniczyły 633 dobrane losowo osoby w wieku 50 lat i więcej (312 Greków oraz 321 Polaków). Narzędziem badawczym był autorski kwestionariusz ankiety.

Wyniki: Na spożycie 4-5 posiłków w ciągu dnia wskazało 41,07\% osób, w tym mniej Greków (31,09\%), niż Polaków (50,78\%). Ryby 1-2 razy w tygodniu były spożywane przez 29,27\% badanych, w tym przez więcej osób z Grecji (23,40\%), niż z Polski (15,26\%). Pełnoziarniste pieczywo kilka razy dzienne było spożywane przez 20,7\% osób, w tym przez 19,55\% Greków oraz 21,81\% Polaków. Kilkukrotne w ciągu tygodnia spożywanie grubych kasz i makaronów zadeklarowało $23,06 \%$ badanych, w tym 25,64\% Greków oraz 20,56\% Polaków.

Wnioski: Zachowania żywieniowe dorosłych mieszkańców Grecji i Polski są zróżnicowane, przy czym korzystniejszymi zachowaniami charakteryzowali się mieszkańcy Grecji. Istnieją znaczne różnice między spożyciem przez mieszkańców Grecji i Polski produktów spożywczych bogatych w błonnik i kwasy tłuszczowe omega-3.

Słowa kluczowe: zachowania żywieniowe, częstość spożycia, Polska, Grecja

Corresponding author: Elżbieta Szczepańska, Ph.D, Department of Human Nutrition, School of Health Sciences in Bytom, Medical University of Silesia, Jordana 19, 41-808 Zabrze, Poland, Phone: +48 32 275-51-97; e-mail: eszczepanska@sum.edu.pl

(C) Copyright by the National Institute of Public Health - National Institute of Hygiene 


\section{INTRODUCTION}

The length and the quality of life are determined by a variety of factors. The greatest importance is attributed to environmental factors, such as proper eating behaviours, an appropriate level of physical activity, a reasonable way of spending free time and getting sufficient sleep [3, 17]. Dietary guidelines for adults are based on providing the body with appropriate amounts of vegetables, fruit, lean meat and dairy products, whole-grain products, as well as fats, mainly of plant origin. Compliance with these recommendations helps prevent a number of diet-related diseases and may increase average life expectancy [ 16 , 20]. The place of residence is associated with certain differences in nutritional culture, with food traditions and customs being of great importance. Furthermore, the location of a given country in a specific climate zone has an impact on the choice of food products and their preparation. However, despite these differences, recommendations for healthy eating patterns in different groups of population, including adults, have been developed based on the body's need for energy and nutrients as well as physiological changes [7].

The ageing of the world population, including Europeans, which has been observed for many years, has led to an increased proportion of older people in their populations [3, 17]. According to EUROSTAT data, older people accounted for $17.1 \%$ of the total Polish population in 2018, which is 3.6\% higher compared to 2008. Greece, on the other hand, is among EU countries with the highest ageing rates. Currently, the proportion of elderly people in Greece is $21.8 \%$ compared to $18.7 \%$ in 2008 . The number of elderly people is growing. It is estimated that they will account for $31.3 \%$ of the European population in 2100 , which is higher by $11.5 \%$ compared to 2018 [5]. Therefore, it seems important to follow well-balanced diet to improve both life expectancy and quality. This should begin already early in life, preferably in childhood.

The aim of the study was to analyse eating behaviours among adult residents of Greece and Poland as well as to investigate to what extent these behaviours are impacted by the place of residence of respondents.

\section{MATERIALS AND METHODS}

A total of 633 randomized respondents at the age of 50 or older, including 312 Greeks and 321 Poles, were included in the study, which was conducted personally in the winter season of 2019. The study used an original questionnaire consisting of 3 parts. The first part inquired about socio-demographic data, the second part was comprised of questions on eating behaviours and the frequency of consuming selected food products during the last 30 days before the study, and the third part was a self-assessment of eating behaviours.

Participation in this study was voluntary. All respondents were informed about the aim of the study and have agreed to participate in it. The study didn't require approval of the Bioethics Committee.

The main study was preceded by a pilot study to verify the research tool, including the elimination of language errors. The pilot study was conducted in a group of 15 respondents. After analysing the obtained answers, corrections were performed so that the questions included in the questionnaire be fully comprehensible to the respondents. This was followed by the main study.

The obtained results were processed using the Microsoft Office Excel 2010. The answers obtained were classified taking into account the place of residence of respondents. Statistica 13.3 (TIBCO Statistica $^{\mathrm{TM}}$ ) was used for statistical analysis. A chisquare test was used to analyse the relationships between the place of residence, eating habits and consumption rates for different groups of food products. The level of statistical significance was set at $\mathrm{p}<0.05$ for all analyses.

\section{RESULTS}

The characteristics of the study group is shown in Table 1.

A total of 633 respondents, including $49.29 \%$ of Greeks and $50.71 \%$ of Poles, were included in the study. Respondents aged between 50 and 55 years dominated, with $33.65 \%$ of Greeks and $13.40 \%$ of Poles in this group. The majority of study participants declared secondary education, with most Greeks reporting primary education (34.94\%) and most Poles declaring secondary education (41.12\%). Most respondents were unemployed, with fewer professionally inactive Greeks $(46.47 \%)$ than Poles (59.81\%). $46.47 \%$ of Greeks and $49.53 \%$ of Poles respondents lived with their spouses in their family home (Table 1).

Selected eating habits are shown in Tables 2-3.

Recommended consumption of 4-5 meals a day was declared by $31.09 \%$ Greeks and $50.78 \%$ Poles. $81 \%$ of Poles and $55.13 \%$ of Greeks had breakfast on a daily basis. Most Greeks (35.26\%) consumed their breakfast 30 minutes after waking up, whereas most Poles (38.94\%) had their breakfast 30-60 minutes after waking up. The last meal was most often consumed 30 - 60 minutes before bedtime, as declared by $28.53 \%$ of Greeks and $27.41 \%$ of Poles (Table 2).

A recommended intake of water was declared by $32.05 \%$ of Greeks and $21.50 \%$ of Poles. Freshly squeezed fruit and vegetable juices were rarely 
Table 1. Characteristics of the study group

\begin{tabular}{|c|c|c|c|c|c|}
\hline \multirow{2}{*}{\multicolumn{2}{|c|}{ Variables }} & \multicolumn{2}{|c|}{ Greece } & \multicolumn{2}{|c|}{ Poland } \\
\hline & & \multirow{2}{*}{$\begin{array}{c}\mathrm{n}=312 \\
181\end{array}$} & \multirow{2}{*}{$\begin{array}{c}\% \\
58.01\end{array}$} & \multirow{2}{*}{$\begin{array}{c}\mathrm{n}=321 \\
198\end{array}$} & \multirow{2}{*}{$\begin{array}{c}\% \\
61.68\end{array}$} \\
\hline $\mathrm{S}$ & women & & & & \\
\hline sex & men & 131 & 41.99 & 123 & 38.32 \\
\hline \multirow{3}{*}{ Age group } & $50-59$ years & 158 & 50.64 & 84 & 26.17 \\
\hline & 60-69 years & 93 & 29.81 & 158 & 49.22 \\
\hline & $\geq 70$ years & 61 & 19.55 & 79 & 24.61 \\
\hline \multirow{4}{*}{ Education } & higher & 17 & 5.45 & 40 & 12.46 \\
\hline & vocational & 89 & 28.53 & 109 & 33.96 \\
\hline & secondary & 97 & 31.09 & 132 & 41.12 \\
\hline & primary & 109 & 34.94 & 40 & 12.46 \\
\hline \multirow{2}{*}{ Professional activity } & working & 167 & 53.53 & 129 & 40.19 \\
\hline & non-working & 145 & 46.47 & 192 & 59.81 \\
\hline \multirow{4}{*}{$\begin{array}{l}\text { Place of residence } \\
\text { and living status }\end{array}$} & in family home, alone & 73 & 23.40 & 82 & 25.55 \\
\hline & in family home, with my husband/wife & 145 & 46.47 & 159 & 49.53 \\
\hline & in multigenerational family home & 80 & 25.64 & 80 & 24.92 \\
\hline & in a nursing home & 14 & 4.49 & 0 & 0.00 \\
\hline
\end{tabular}

Table 2. Nutritional behaviours concerning meals consumption

\begin{tabular}{|c|c|c|c|c|c|c|}
\hline \multirow{2}{*}{\multicolumn{2}{|c|}{ Selected nutritional behaviour }} & \multicolumn{2}{|c|}{ Greece } & \multicolumn{2}{|c|}{ Poland } & \multirow{2}{*}{$\begin{array}{c}\text { Statistical } \\
\text { analysis }\end{array}$} \\
\hline & & $\mathrm{n}=312$ & $\%$ & $\mathrm{n}=321$ & $\%$ & \\
\hline \multirow{3}{*}{ Amount of meals } & 3 or less meals & 196 & 62.82 & 139 & 43.30 & \multirow{3}{*}{$\begin{array}{c}\mathrm{p}<0.05 \\
\mathrm{Vc}=0.20\end{array}$} \\
\hline & $4-5$ meals & 97 & 31.09 & 163 & 50.78 & \\
\hline & more than 5 meals & 19 & 6.09 & 19 & 5.92 & \\
\hline \multirow{3}{*}{$\begin{array}{l}\text { Consumption of } \\
\text { breakfast }\end{array}$} & yes, sometimes & 99 & 31.73 & 53 & 16.51 & \\
\hline & yes, always & 172 & 55.13 & 260 & 81.00 & \\
\hline & no, never & 41 & 13.14 & 8 & 2.49 & \\
\hline \multirow{4}{*}{$\begin{array}{l}\text { Time of eating the first } \\
\text { meal (after waking up) }\end{array}$} & up to 30 minutes & 110 & 35.26 & 120 & 37.38 & \multirow{4}{*}{$\begin{array}{l}\mathrm{p}=0.018 \\
\mathrm{Vc}=0.13\end{array}$} \\
\hline & $30-60$ minutes & 98 & 31.41 & 125 & 38.94 & \\
\hline & $>60$ minutes & 42 & 13.46 & 39 & 12.15 & \\
\hline & variously & 62 & 19.87 & 37 & 11.53 & \\
\hline \multirow{5}{*}{$\begin{array}{l}\text { Time of eating the last } \\
\text { meal (before bedtime) }\end{array}$} & immediately before & 16 & 5.13 & 32 & 9.97 & \multirow{5}{*}{ ND* } \\
\hline & $30-60$ minutes & 89 & 28.53 & 88 & 27.41 & \\
\hline & 60-120 minutes & 81 & 25.96 & 72 & 22.43 & \\
\hline & $>120$ minutes & 70 & 22.44 & 78 & 24.30 & \\
\hline & variously & 56 & 17.95 & 51 & 15.89 & \\
\hline
\end{tabular}

*ND - inability to run statistical analysis due to small group size

consumed by the respondents. $48.4 \%$ and $91.35 \%$ of Greeks, $64.8 \%$ and $91.28 \%$ of Poles and $91.31 \%$ did not drink fruit or vegetable juices, respectively. The majority of Polish respondents had 2 glasses of tea $(35.83 \%)$ and up to one glass of coffee $(38.01 \%)$ per day. The majority of Greek respondents declared that they had no tea $(42.95 \%)$ and 2 glasses of coffee (39.74\%) daily (Table 3).

Consumption rates for the selected groups of food products are shown in Tables 4-7.

Whole wheat bread was consumed several times daily by $19.55 \%$ of Greeks and $21.81 \%$ of Poles. Both Greeks and Poles consumed white bread more often during the day, $25.00 \% 30.22 \%$ respectively. Coarse groats and pasta were consumed a few times weekly by $25.64 \%$ of Greeks and $20.56 \%$ of Poles (Table 4 ).

Daily consumption of cow's milk was declared by more Greeks (37.18\%) than Poles (25.86\%); whereas daily consumption of fermented dairy beverages was declared by $27.41 \%$ Poles and $12.82 \%$ Greeks. Cottage cheese was consumed a few times weekly by more Greeks than Poles $(41.35 \%$ vs. $31.15 \%$, respectively). Cheese and processed cheese were consumed several times weekly by $43.61 \%$ of Poles and $39.10 \%$ of Greeks (Table 5). 
Table 3. Nutritional behaviours concerning fluids intake

\begin{tabular}{|c|c|c|c|c|c|c|}
\hline \multirow{2}{*}{\multicolumn{2}{|c|}{ Selected nutritional behaviour }} & \multicolumn{2}{|c|}{ Greece } & \multicolumn{2}{|c|}{ Poland } & \multirow{2}{*}{$\begin{array}{c}\text { Statistical } \\
\text { analysis }\end{array}$} \\
\hline & & \multirow{2}{*}{$\begin{array}{c}\mathrm{n}=312 \\
100\end{array}$} & \multirow{2}{*}{$\begin{array}{c}\% \\
32.05\end{array}$} & \multirow{2}{*}{$\begin{array}{c}\mathrm{n}=321 \\
69\end{array}$} & \multirow{2}{*}{\begin{tabular}{c|}
$\%$ \\
21.50
\end{tabular}} & \\
\hline \multirow{4}{*}{ Water intake } & 6 glasses or more & & & & & \multirow{4}{*}{$\begin{aligned} \mathrm{p} & <0.5 \\
\mathrm{Vc} & =0.15\end{aligned}$} \\
\hline & 4-5 glasses (cups?) & 108 & 34.62 & 113 & 35.20 & \\
\hline & 3 glasses or less & 93 & 29.81 & 113 & 35.20 & \\
\hline & I don't drink water & 11 & 3.53 & 26 & 8.10 & \\
\hline \multirow{3}{*}{$\begin{array}{l}\text { Freshly squeezed fruit } \\
\text { juices intake }\end{array}$} & 2 glasses or more & 12 & 3.85 & 21 & 6.54 & \multirow{3}{*}{$\begin{array}{c}\mathrm{p}<0.05 \\
\mathrm{Vc}=0.20\end{array}$} \\
\hline & 1 glass or less & 149 & 47.76 & 92 & 28.66 & \\
\hline & $\begin{array}{l}\text { I don't drink fruit } \\
\text { juices }\end{array}$ & 151 & 48.40 & 208 & 64.80 & \\
\hline \multirow{3}{*}{$\begin{array}{l}\text { Freshly squeezed } \\
\text { vegetable juices intake }\end{array}$} & 2 glasses or more & 9 & 2.88 & 6 & 1.87 & \multirow{3}{*}{$\mathrm{ND} *$} \\
\hline & 1 glass or less & 18 & 5.77 & 22 & 6.85 & \\
\hline & $\begin{array}{l}\text { I don't drink vegetable } \\
\text { juices }\end{array}$ & 285 & 91.35 & 293 & 91.28 & \\
\hline \multirow{4}{*}{ Tea } & 3 glasses or more & 20 & 6.41 & 105 & 32.71 & \multirow{4}{*}{$\begin{array}{l}\mathrm{p}<0.05 \\
\mathrm{Vc}=0.50\end{array}$} \\
\hline & 2 glasses & 42 & 13.46 & 115 & 35.83 & \\
\hline & 1 glass or less & 116 & 37.18 & 61 & 19.00 & \\
\hline & I don't drink tea & 134 & 42.95 & 40 & 12.46 & \\
\hline \multirow{4}{*}{ Coffee } & 3 glasses or more & 80 & 25.64 & 49 & 15.26 & \multirow{4}{*}{$\begin{array}{l}\mathrm{p}<0.05 \\
\mathrm{Vc}=0.17\end{array}$} \\
\hline & 2 glasses & 124 & 39.74 & 113 & 35.20 & \\
\hline & 1 glass or less & 86 & 27.56 & 122 & 38.01 & \\
\hline & I don't drink coffee & 22 & 7.05 & 37 & 11.53 & \\
\hline
\end{tabular}

* ND - inability to run statistical analysis due to small group size

Table 4. Consumption frequency of cereal products

\begin{tabular}{|c|c|c|c|c|c|c|}
\hline \multirow{2}{*}{\multicolumn{2}{|c|}{ Consumption frequency }} & \multicolumn{2}{|c|}{ Greece } & \multicolumn{2}{|c|}{ Poland } & \multirow{2}{*}{$\begin{array}{c}\text { Statistical } \\
\text { analysis }\end{array}$} \\
\hline & & \multirow{2}{*}{$\begin{array}{c}n=312 \\
61\end{array}$} & \multirow{2}{*}{$\begin{array}{c}\% \\
19.55 \\
\end{array}$} & \multirow{2}{*}{$\begin{array}{c}n=321 \\
70\end{array}$} & \multirow{2}{*}{$\begin{array}{c}\% \\
21.81\end{array}$} & \\
\hline \multirow{6}{*}{ Whole wheat bread } & a couple times a day & & & & & \multirow{6}{*}{$\begin{array}{c}\mathrm{p}<0.05 \\
\mathrm{Vc}=0.19\end{array}$} \\
\hline & once a day & 44 & 14.10 & 71 & 22.12 & \\
\hline & a couple times a week & 59 & 18.91 & 35 & 10.90 & \\
\hline & once a week or less & 23 & 7.37 & 44 & 13.71 & \\
\hline & occasionally & 32 & 10.26 & 33 & 10.28 & \\
\hline & never & 93 & 29.81 & 68 & 21.18 & \\
\hline \multirow{6}{*}{ White bread } & a couple times a day & 78 & 25.00 & 97 & 30.22 & \multirow{6}{*}{$\begin{array}{c}\mathrm{p}=0.04 \\
\mathrm{Vc}=0.14\end{array}$} \\
\hline & once a day & 67 & 21.47 & 76 & 23.68 & \\
\hline & a couple times a week & 46 & 14.74 & 45 & 14.02 & \\
\hline & once a week or less & 20 & 6.41 & 29 & 9.03 & \\
\hline & occasionally & 26 & 8.33 & 29 & 9.03 & \\
\hline & never & 75 & 24.04 & 45 & 14.02 & \\
\hline \multirow{5}{*}{$\begin{array}{l}\text { Coarse groats and } \\
\text { pasta }\end{array}$} & a couple times a day & 26 & 8.33 & 10 & 3.12 & \multirow{5}{*}{$\begin{array}{c}\mathrm{p}<0.05 \\
\mathrm{Vc}=0.33\end{array}$} \\
\hline & once a day & 80 & 25.64 & 66 & 20.56 & \\
\hline & a couple times a week & 70 & 22.44 & 158 & 49.22 & \\
\hline & once a week or less & 29 & 9.29 & 42 & 13.08 & \\
\hline & occasionally & 107 & 34.29 & 45 & 14.02 & \\
\hline
\end{tabular}


Table 5. Consumption frequency of dairy products

\begin{tabular}{|c|c|c|c|c|c|c|}
\hline \multirow{2}{*}{\multicolumn{2}{|c|}{ Consumption frequency }} & \multicolumn{2}{|c|}{ Greece } & \multicolumn{2}{|c|}{ Poland } & \multirow{2}{*}{$\begin{array}{l}\text { Statistical } \\
\text { analysis }\end{array}$} \\
\hline & & \multirow{2}{*}{$\begin{array}{c}\mathrm{n}=312 \\
116\end{array}$} & \multirow{2}{*}{$\begin{array}{c}\% \\
37.18 \\
\end{array}$} & \multirow{2}{*}{$\begin{array}{c}\mathrm{n}=321 \\
83\end{array}$} & \multirow{2}{*}{$\begin{array}{c}\% \\
25.86 \\
\end{array}$} & \\
\hline \multirow{4}{*}{ Cow's milk } & daily & & & & & \multirow{4}{*}{$\begin{array}{c}\mathrm{p}<0.05 \\
\mathrm{Vc}=0.17\end{array}$} \\
\hline & a couple times a week & 43 & 13.78 & 83 & 25.86 & \\
\hline & once a week or less & 46 & 14.74 & 43 & 13.40 & \\
\hline & occasionally or never & 107 & 34.29 & 112 & 34.89 & \\
\hline \multirow{4}{*}{ Sheep's milk } & daily & 19 & 6.09 & 3 & 0.93 & \multirow{4}{*}{ ND* } \\
\hline & a couple times a week & 18 & 5.77 & 3 & 0.93 & \\
\hline & once a week or less & 33 & 10.58 & 0 & 0.00 & \\
\hline & occasionally or never & 242 & 77.56 & 315 & 98.13 & \\
\hline \multirow{4}{*}{$\begin{array}{l}\text { Fermented dairy } \\
\text { beverages }\end{array}$} & daily & 40 & 12.82 & 88 & 27.41 & \multirow{4}{*}{$\begin{array}{c}\mathrm{p}<0.05 \\
\mathrm{Vc}=0.23\end{array}$} \\
\hline & a couple times a week & 94 & 30.13 & 113 & 35.20 & \\
\hline & once a week or less & 70 & 22.44 & 59 & 18.38 & \\
\hline & occasionally or never & 108 & 34.62 & 61 & 19.00 & \\
\hline \multirow{4}{*}{ Cottage cheese } & daily & 71 & 22.76 & 34 & 10.59 & \multirow{4}{*}{$\begin{array}{c}\mathrm{p}<0.05 \\
\mathrm{Vc}=0.26\end{array}$} \\
\hline & a couple times a week & 129 & 41.35 & 100 & 31.15 & \\
\hline & once a week or less & 61 & 19.55 & 131 & 40.81 & \\
\hline & occasionally or never & 51 & 16.35 & 56 & 17.45 & \\
\hline \multirow{4}{*}{$\begin{array}{l}\text { Cheese and processed } \\
\text { cheese }\end{array}$} & daily & 58 & 18.59 & 36 & 11.21 & \multirow{4}{*}{$\begin{array}{l}\mathrm{p}=0.005 \\
\mathrm{Vc}=0.14\end{array}$} \\
\hline & a couple times a week & 122 & 39.10 & 140 & 43.61 & \\
\hline & once a week or less & 72 & 23.08 & 100 & 31.15 & \\
\hline & occasionally or never & 60 & 19.23 & 45 & 14.02 & \\
\hline
\end{tabular}

* ND - inability to run statistical analysis due to small group size

Fish was consumed at 1-2 servings a week by more Greeks (23.40\%) than Poles (15.26\%). Sea food was consumed several times a week by more Greeks (12.82\%) than Poles (1.25\%). White meat was

consumed several times a week by $45.51 \%$ of Greeks and $49.53 \%$ of Poles, whereas the same rate for red meat was $32.37 \%$ for Greeks and $31.78 \%$ for Poles. (Table 6).

Table 6. Consumption frequency of meat, fish and seafoods

\begin{tabular}{|c|c|c|c|c|c|c|}
\hline \multirow{2}{*}{\multicolumn{2}{|c|}{ Consumption frequency }} & \multicolumn{2}{|c|}{ Greece } & \multicolumn{2}{|c|}{ Poland } & \multirow{2}{*}{$\begin{array}{c}\text { Statistical } \\
\text { analysis }\end{array}$} \\
\hline & & $\mathrm{n}=312$ & $\%$ & $\mathrm{n}=321$ & $\%$ & \\
\hline \multirow{4}{*}{ Fish } & daily & 13 & 4.17 & 5 & 1.56 & \multirow{4}{*}{$\begin{array}{c}\mathrm{p}<0.05 \\
\mathrm{Vc}=0.19\end{array}$} \\
\hline & a couple times a week & 73 & 23.40 & 49 & 15.26 & \\
\hline & once a week or less & 196 & 62.82 & 201 & 62.62 & \\
\hline & occasionally or never & 30 & 9.62 & 66 & 20.56 & \\
\hline \multirow{4}{*}{$\begin{array}{l}\text { Seafood (lobster, crab, } \\
\text { prawns, langoustine) }\end{array}$} & daily & 10 & 3.21 & 0 & 0.00 & \multirow{4}{*}{ ND* } \\
\hline & a couple times a week & 40 & 12.82 & 4 & 1.25 & \\
\hline & once a week or less & 126 & 40.38 & 15 & 4.67 & \\
\hline & occasionally or never & 136 & 43.59 & 302 & 94.08 & \\
\hline \multirow{4}{*}{$\begin{array}{l}\text { White meat } \\
\text { (chicken, turkey) }\end{array}$} & daily & 16 & 5.13 & 31 & 9.66 & \multirow{4}{*}{ ND* } \\
\hline & a couple times a week & 142 & 45.51 & 159 & 49.53 & \\
\hline & once a week or less & 140 & 44.87 & 118 & 36.76 & \\
\hline & occasionally or never & 14 & 4.49 & 13 & 4.05 & \\
\hline \multirow{4}{*}{$\begin{array}{l}\text { Red meat } \\
\text { (pork, beef) }\end{array}$} & daily & 19 & 6.09 & 17 & 5.30 & \multirow{4}{*}{$\begin{array}{c}\mathrm{p}=0.04 \\
\mathrm{Vc}=0.11\end{array}$} \\
\hline & a couple times a week & 101 & 32.37 & 102 & 31.78 & \\
\hline & once a week or less & 163 & 52.24 & 148 & 46.11 & \\
\hline & occasionally or never & 29 & 9.29 & 54 & 16.82 & \\
\hline
\end{tabular}

* ND - inability to run statistical analysis due to small group size 
Potatoes were consumed several times a week by fruit was declared by more Poles $(67.60 \%)$ than Greeks $57.69 \%$ of Greeks and $56.39 \%$ of Poles. Daily vegetable (56.73\%). Daily use of olive oil was reported by more consumption was declared by more Greeks than Poles Greeks (68.59\%) than Poles (12.15\%). Unsalted nuts (52.88\% and $34.89 \%$, respectively). Legume seeds were consumed several times weekly by more Greeks were consumed several times a week by more Greeks $\quad(24.68 \%)$ than Poles (7.48\%) (Table 7). $(30.13 \%)$ than Poles $(5.92 \%)$. Daily consumption of

Table 7. Consumption frequency of vegetables, fruits, nuts and olives

\begin{tabular}{|c|c|c|c|c|c|c|}
\hline \multirow{2}{*}{\multicolumn{2}{|c|}{ Consumption frequency }} & \multicolumn{2}{|c|}{ Greece } & \multicolumn{2}{|c|}{ Poland } & \multirow{2}{*}{$\begin{array}{c}\text { Statistical } \\
\text { analysis }\end{array}$} \\
\hline & & $\mathrm{n}=312$ & $\%$ & $\mathrm{n}=321$ & $\%$ & \\
\hline \multirow{4}{*}{ Potatoes } & daily & 31 & 9.94 & 98 & 30.53 & \multirow{4}{*}{$\begin{array}{c}\mathrm{p}<0.05 \\
\mathrm{Vc}=0.30\end{array}$} \\
\hline & a couple times a week & 180 & 57.69 & 181 & 56.39 & \\
\hline & once a week or less & 71 & 22.76 & 30 & 9.35 & \\
\hline & occasionally or never & 30 & 9.62 & 12 & 3.74 & \\
\hline \multirow{4}{*}{ Vegetables } & daily & 165 & 52.88 & 112 & 34.89 & \multirow{4}{*}{$\begin{array}{c}\mathrm{p}<0.05 \\
\mathrm{Vc}=0.21\end{array}$} \\
\hline & a couple times a week & 96 & 30.77 & 164 & 51.09 & \\
\hline & once a week or less & 29 & 9.29 & 27 & 8.41 & \\
\hline & occasionally or never & 22 & 7.05 & 18 & 5.61 & \\
\hline \multirow{4}{*}{ Pulses } & daily & 33 & 10.58 & 5 & 1.56 & \multirow{4}{*}{ ND* } \\
\hline & a couple times a week & 94 & 30.13 & 19 & 5.92 & \\
\hline & once a week or less & 156 & 50.00 & 118 & 36.76 & \\
\hline & occasionally or never & 29 & 9.29 & 179 & 55.76 & \\
\hline \multirow{4}{*}{ Fruits } & daily & 177 & 56.73 & 217 & 67.60 & \multirow{4}{*}{$\begin{array}{l}\mathrm{p}=0.042 \\
\mathrm{Vc}=0.11\end{array}$} \\
\hline & a couple times a week & 80 & 25.64 & 65 & 20.25 & \\
\hline & once a week or less & 32 & 10.26 & 23 & 7.17 & \\
\hline & occasionally or never & 23 & 7.37 & 16 & 4.98 & \\
\hline \multirow{4}{*}{ Unsalted nuts } & daily & 41 & 13.14 & 14 & 4.36 & \multirow{4}{*}{$\begin{array}{c}\mathrm{p}<0.05 \\
\mathrm{Vc}=0.40\end{array}$} \\
\hline & a couple times a week & 77 & 24.68 & 24 & 7.48 & \\
\hline & once a week or less & 79 & 25.32 & 41 & 12.77 & \\
\hline & occasionally or never & 115 & 36.86 & 242 & 75.39 & \\
\hline \multirow{4}{*}{ Olives/olive oil } & daily & 214 & 68.59 & 39 & 12.15 & \multirow{4}{*}{$\begin{array}{c}\mathrm{p}<0.05 \\
\mathrm{Vc}=0.60\end{array}$} \\
\hline & a couple times a week & 59 & 18.91 & 107 & 33.33 & \\
\hline & once a week or less & 27 & 8.65 & 64 & 19.94 & \\
\hline & occasionally or never & 12 & 3.85 & 111 & 34.58 & \\
\hline
\end{tabular}

* ND - inability to run statistical analysis due to small group size

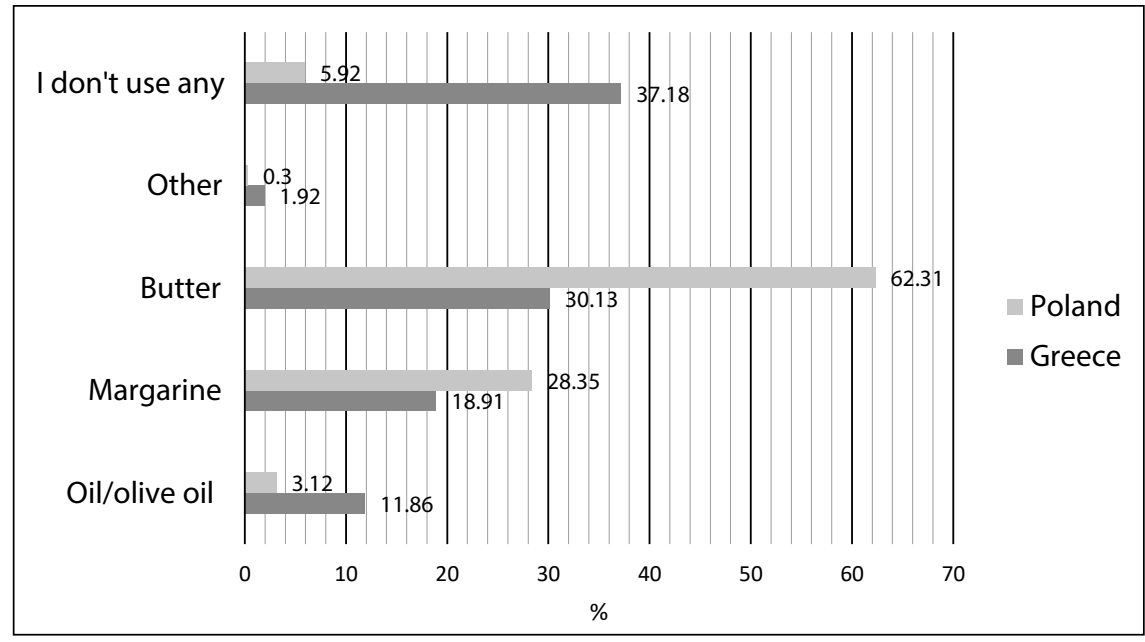

Figure 1. Fats used to spread on bread 
Types of fat used for bread and food processing are shown in Figures 1 and 2.

Butter was the most common fat for bread with more Poles (62.31\%) than Greeks (30.13\%) using this product. Oil and olive oil were the most common fats used for food processing and were more popular with Greeks (91.03\%) than Poles (67.29\%) (Figures 1 and 2).

The majority of respondents self-assessed their eating habits as good, including $55.45 \%$ of Greeks and $56.39 \%$ of Poles (Figure 3).

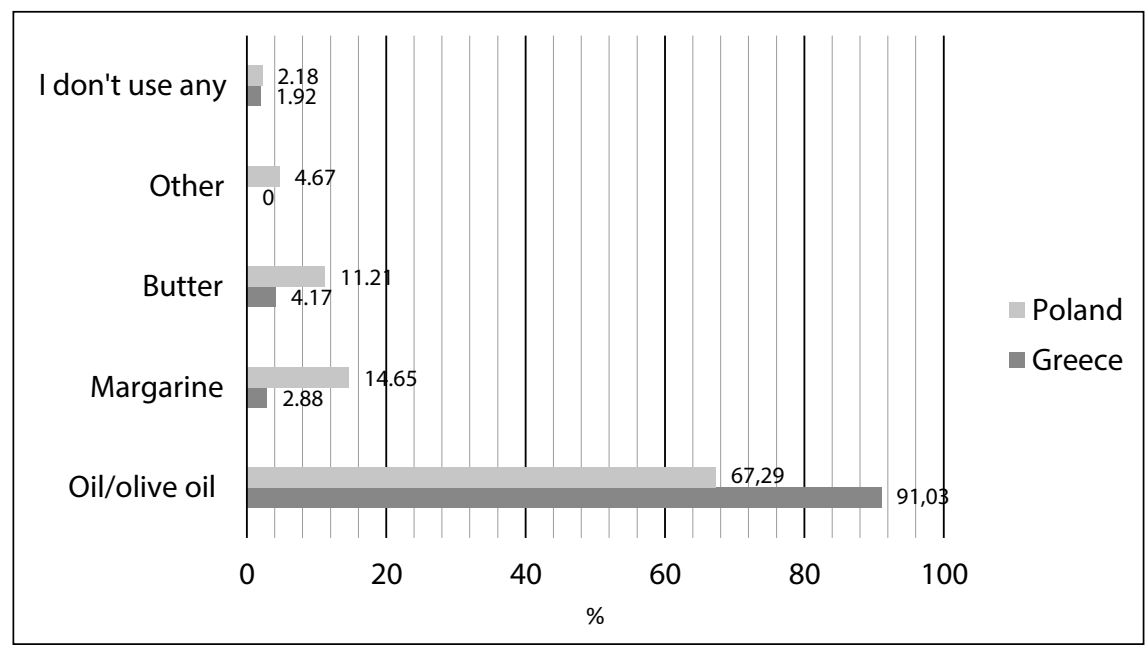

Figure 2. Fats used for food processing

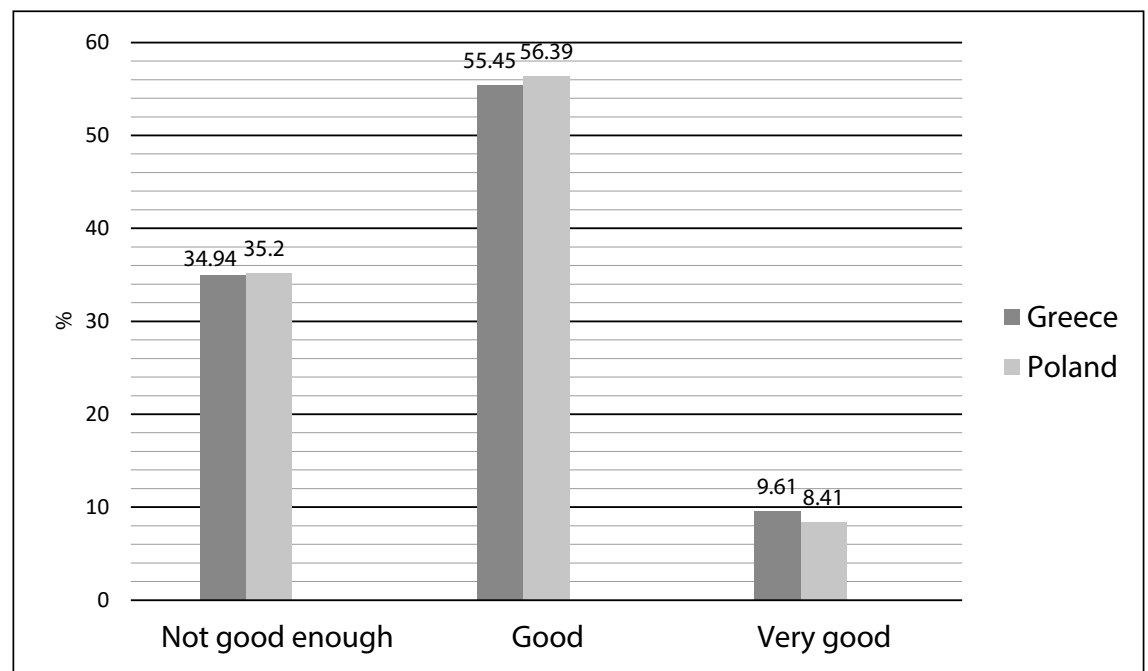

Figure 3. Self-assessment of eating habits of the study group

\section{DISCUSSION}

Over the past few decades, technological development, including advances in medicine, and better living conditions have led to an improvement of both life quality and expectancy. Great importance is also attributed to eating habits, which should depend on, among other things, age, sex and type of physical activity.

Regular meals consumption reduces the risk of overweight, obesity as well as occurrence of metabolic diseases. The study showed that the recommended intake of $4-5$ meals a day was declared by $41.07 \%$ of respondents, including less Greeks (31.09\%) than Poles (50.78\%). Different results were obtained by JeruszkaBielak et al. [10] who conducted their study in 1,144 respondents aged 65-79 years, who came from five European countries: France, Italy, the Netherlands, Poland and the United Kingdom. The authors showed that only $16.9 \%$ of respondents had more than 3 meals daily, whereas the vast majority of respondents $(69.3 \%)$ declared 3 meals a day. The present study showed that daily consumption of breakfast was declared by $68.25 \%$ of respondents, including more Poles $(81 \%)$ than Greeks (55.13\%). More optimistic results were reported by the authors investigating eating habits among Chileans over the age of 60 years. Most of them (76.6\%) reported daily consumption of breakfast [4].

Our study demonstrated that the daily intake of tea was usually 1 glass or less $(27.96 \%)$, or none $(27.49 \%)$. 
However, a comparative analysis showed a significant variation: most Greeks declared that they consumed no tea $(42.95 \%)$ or up to 1 glass of tea daily $(37.18 \%)$, whereas most Poles declared having 2 to 3 glasses of tea daily (35.83\% and $32.71 \%$, respectively). Similar findings were reported by Naumovski et al. [15] who investigated the intake of green and black tea among Greeks aged $\geq 50$ years in the context of ageing. The authors showed that the respondents usually had one cup of tea or less (both black and green) a day. Furthermore, the study confirmed that green tea components play an important role in successful ageing, as well as are key elements of healthy diet in adults.

Our study demonstrated that wholemeal bread was consumed several times daily less frequently than white bread, i.e. $20.7 \%$ and $27.65 \%$, respectively, and that coarse groats and pasta were consumed a few times weekly by $23.06 \%$ of respondents. Different results were presented by Suliga [18], who assessed health-related eating behaviours in adult and elderly individuals. According to the author, $17.7 \%$ of the 166 respondents declared consumption of wholemeal bread and coarse groats several times daily (including $16.6 \%$ of women and $19.4 \%$ of men), whereas $15.9 \%$ of respondents (14.4\% of women and $17.9 \%$ of men) reported that they consumed these products several times a week. Inadequate consumption of whole grains may be associated with the occurrence of various civilization diseases, such as diabetes, cardiovascular diseases and certain cancers [11]. Additionally, insufficient amount of fibre in the diet of which the best source are whole grains, may result in greater consumption of products of low nutritional value that could lead to overweight or obesity.

Milk and dairy products should be an important element of a daily diet due to their content of calcium, which is crucial for bone mineral density [14]. Our study showed that $31.44 \%$ of respondents declared daily intake of cow's milk, and $20.22 \%$ of respondents reported daily intake of fermented dairy beverages. Foscolou et al. [6], who conducted a study to assess the relationship between whole grain consumption and ageing, considering general eating habits of adult residents of the Mediterranean region, found that the intake of dairy products was 3.9 servings a week. Different findings were obtained by Hassapidou et al. [8] who conducted their study among 4,026 Greeks to assess the impact of Mediterranean diet on weight loss in the study population. The authors showed that the respondents in the study group reported 8.7 servings of these products weekly. Furthermore, the authors of the study estimated that the mean weekly intake of fish was 4.9 servings, and that the same intake of poultry and red meat was 1.5 and 13.4 portions, respectively. Adequate consumption of meat and fish provides many essential nutrients, including complete protein which has positive effects on the body. Fish are also a good source of polyunsaturated fats, which support the proper functioning of the cardiovascular system. Insufficient amount of this products in the diet might result in occurrence of many diseases [13]. In our study, $29.7 \%$ of respondents declared an intake of $1-2$ servings of fish per week; $47.55 \%$ of respondents consumed white meat several times weekly; and $32.07 \%$ of respondents consumed red meat several times weekly. Different findings were presented by Suliga [18], who reported fish intake several times a week in $17.5 \%$ of respondents (including $15.3 \%$ of women and $20.6 \%$ of men), and the highest intake rates of meat and cold meats among respondents who consumed these products several times daily (36.8\%), with significantly higher rates among men $(52.9 \%)$ compared to women $(25.5 \%)$.

Vegetables, fruits and dry legume seeds play an important role in human nutrition as they are a source of multiple vitamins and minerals, dietary fibre, as well as bioactive substances, which are beneficial for the body. Therefore, they play a key role in preventing cardiovascular diseases, gastric, oesophageal, pancreatic and colon cancer, as well as overweight and obesity [2]. In our study, daily consumption of vegetables was declared by $43.76 \%$ of respondents (it was higher among Greeks than Poles), whereas daily consumption of fruit was reported by $62.24 \%$ of study participants (higher among Poles than Greeks). Legume seeds were consumed several times a week by only $17.85 \%$ of respondents. Different results were obtained by Kossioni and Bellou [12] who reported daily fruit intake in $86.9 \%$ of respondents over 60 years of age. Hernández-Galiot and Goñi [9] showed in their study that $44.2 \%$ of women and $47.2 \%$ of men consumed at least two servings of vegetables $(1$ serving $=200 \mathrm{~g})$ a day. At least three portions of fruit, including fruit juices, per day were consumed by $55.8 \%$ of women and $52.8 \%$ of men. At least three servings (1 serving $=150 \mathrm{~g})$ of legume seeds were consumed weekly by $41.9 \%$ of women and $41.7 \%$ of men.

Regular intake of nuts, which are a source of polyunsaturated fatty acids, may significantly improve lipid profile and, consequently, reduce the risk of myocardial infarction, stroke and cardiovascular death [7]. Our study showed that unsalted nuts were consumed several times weekly by $15.96 \%$ of respondents, with higher rates in Greeks $(24.68 \%)$ than in Poles (7.48\%). Hernández-Galiot and Goñi [9] demonstrated that consumption of one serving of nuts $(30 \mathrm{~g})$ more than once a week was declared by $20.9 \%$ of women and $16.7 \%$ of men. Adamska et al. [1] who assessed eating habits and preferences in different age groups (237 adults) showed that the nut intake rates of 1 serving per week dropped to two times a month. 
According to the authors, the observed tendency may result from dental deterioration in the elderly, which may account for the low nut intake in this group.

To conclude, our study indicates that there are differences in eating habits between Greeks and Poles. Greeks consume more vegetables, nuts, legumes, sea food, fish and olive oil compared to Poles. Their diet is higher in products rich in fibre and omega-3 fatty acids, which has beneficial effects on daily food ration. Studies show that the Mediterranean diet recommended by experts, which is high in vegetables, fruit, olive oil, fish and whole grain products, contributes to reduced risk of cardiovascular incidents, prevents cognitive impairment and, consequently, reduces mortality [9]. Therefore, it seems reasonable to promote this type of diet in all population groups due to multiple potential benefits.

\section{CONCLUSIONS}

There are differences in eating behaviours between Poles and Greeks, with healthier eating habits in the latter group.

There are significant differences between the intake of food products rich in fibre (vegetables, fruit, wholemeal bread, coarse groats and pasta) and omega-3 fatty acids (vegetable oils, unsalted nuts, fish) by residents of Greece and Poland.

\section{Conflict of interest}

The authors declare no conflict of interest.

\section{REFERENCES}

1. Adamska E., Ostrowska L., Adamska E., Maliszewska K., Citko., Waszczeniuk M., Przystupa W., Majeski R., Wasilewska., Milewski R., Krytowski., Górska M.: Różnice w nawykach i preferencjach żywieniowych osób dorosłych w zależności od wieku [Differences in dietary habits and food preferences of adults depending on the age]. Rocz Panstw Zakl Hig 2012;63(1):73-81 (in Polish).

2. Aune D., Giovannucci E., Boffetta P., Fadnes LT., Keum N., Norat T., Greenwood D.C., Riboli E., Vatten L.J., Tonstad S.: Fruit and vegetable intake and the risk of cardiovascular disease, total cancer and all-cause mortality - a systematic review and dose-response meta-analysis of prospective studies. Int. J. Epidemiol. 2017;46(3):1029-1056.

3. Bartoszek A., Nowicki G., Kocka K., Ślusarska B., Bartoszek A., Łuczyk M., Deluga A., Zielonka E.: Health behaviors of people over the age of 65 residing in the home environment, Journal of Education. Health and Sport 2017;7(11):110-128.

4. Durán Agüero S., Priscila Candia P,. Pizarro Mena R.: Validación de contenido de la Encuesta de Calidad de Alimentación del Adulto Mayor (ECAAM) [Content validity of Food Quality Survey of Elderly (FQSE)]. Nutr Hosp. 2017;34(6):1311-1318 (in Spanish).

5. EUROSTAT. https://ec.europa.eu/eurostat/statisticsexplained/index.php/Population_structure_and ageing?fbclid=IwAR3KuRRgxih-2E_UyWoE8ke-6y $\overline{5}$ b9WejDs3xbiCzuiGqVpvK46hXzmmxqXQ. Available (Accessed: 03.10.2019).

6. Foscolou A., D'Cunha NM., Naumovski N., Tyrovolas S., Chrysohoou C., Rallidid L., Matalas A.L., Sidossis L.S., Panagiotakos D.: The Association between Whole Grain Products Consumption and Successful Aging: A Combined Analysis of MEDIS and ATTICA Epidemiological Studies. Nutrients 2019;11:1221.

7. Guasch-Ferré M., Liu X., Malik VS., Sun Q., Willett W.C., Manson J.E., Rexrode K.M., Li Y., Hu F.F., Bhupathiraju S.N.: Nut Consumption and Risk of Cardiovascular Disease. Journal of the American College of Cardiology 2017;25:19-32.

8. Hassapidou M., Tziomalos K., Lazaridou S., Pagkalos I., Papadimitriou K., Kokkinopoulou A., Tzotzas T: The Nutrition Health Alliance (NutriHeAl) Study: A Randomized, Controlled, Nutritional Intervention Based on Mediterranean Diet in Greek Municipalities. J Am Coll Nutr. 2019;1-7.

9. Hernández-Galiot A., Goñi I.: Adherence to the Mediterranean diet pattern, cognitive status and depressive symptoms in an elderly non-institutionalized population. Nutr Hosp 2017;34:338-344.

10.Jeruszka-Bielak M., Kollajtis-Dolowy A., Santoro A, Ostan R., Berendsen A.A.M., Jennings A Meunier N., Marseglia A., Caumon E., Gillings R., de Groot LCPGM, Franceschi C., Hieke S., Pietruszka B.: Are NutritionRelated Knowledge and Attitudes Reflected in Lifestyle and Health Among Elderly People? A Study Across Five European Countries. Front Physiol. 2018;9:994.

11. Kołodziejczyk P., Michniewicz J.: Ziarno zbóż i produkty zbożowe jako źródło błonnika pokarmowego [Cereal grains and cereal products as sources of dietary fibre]. ŻYWNOŚĆ. Nauka. Technologia. Jakość 2018;25,3(116):5-22 (in Polish).

12.Kossioni A., Bellou O.: Eating habits in older people in Greece: the role of age, dental status and chewing difficulties. Arch Gerontol Geriatr. 2011;52(2):197-201.

13. Michalsa G., Nowachowicz J., Bucek T., Wasilewski P.D., Kmiecik M.: Spożycie artykułów żywnościowych $\mathrm{z}$ udziałem mięsa i jego przetworów [Consumption of food products with the participation of meat and meat products] Przegląd Hodowlany 2013;81(6):12-14 (in Polish).

14.Moschonis G., Katsaroli I., Lyritis GP., Manios Y.: The effects of a 30-month dietary intervention on bone mineral density: the Postmenopausal Health Study. Br. J. Nutr. 2010;104(1):100-107.

15. Naumovski N., Foscolou A., D’Cunha N.M., Tyrovolas S., Chrysohoou C., Sidossis L.S., Rallidis L., Matalas A.L., Polychronopoulos E., Pitsavos C., Panagiotakos D.: The Association between Green and Black Tea Consumption on Successful Aging: A Combined Analysis of the ATTICA and MEDiterranean ISlands (MEDIS) Epidemiological Studies. Molecules 2019;24:1862. 
16. Raparelli V., Romiti GF., Spugnardi V., Borgi M., Cangemi R., Basili S., Proietti M., The Eva Collaborative Group: Gender-Related Determinants of Adherence to the Mediterranean Diet in Adults with Ischemic Heart Disease. Nutrients. 2020;12(3):759.

17. Skotnicka M., Gierczak A.: Odżywianie i aktywność fizyczna gwarantem dobrego zdrowia osób starszych [Nutrition and physical activity as the guarantee of the senior's good health]. Zeszyty Naukowe Akademii Morskiej w Gdyni 2016;93:196-202 (in Polish).

18. Suliga E.: Zachowania zdrowotne związane ż̇ywieniem osób dorosłych i starszych [Health behaviours related to the nutrition of adults and elderly people]. Hygeia Public Health 2010;45(1):44-48 (in Polish).
19. Śmidowicz A., Reguła J.: Analiza zachowań żywieniowych predysponujących do rozwoju chorób żywieniowozależnych u osób po 40. roku życia [Analysis of dietary behaviours predisposing to the development of diet-dependent diseases in people over 40 years of age.]. Forum Zaburzeń Metabolicznych 2016;7(1):44-50 (in Polish).

20.Śmidowicz A.: Kulturowe uwarunkowania zachowań żywieniowych [Cultural conditions of nutrition behaviour]. Kosmetologia Estetyczna 2016;2:163-168 (in Polish).

Received: 11.03.2020

Accepted: 08.04.2020 